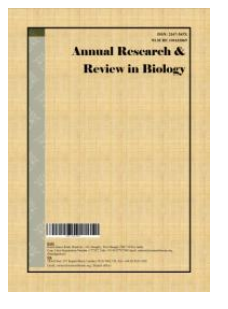

\title{
Foods Causing Highest IgG Immune Response in
} Saudi Arabia

\author{
Abdulhameed F. Alkhateeb ${ }^{1^{*}}$ \\ ${ }^{1}$ Biomedical Engineering, Electrical \& Computer Engineering Department, Faculty of Engineering, \\ King Abdulaziz University, Jeddah, Saudi Arabia.
}

Author's contribution

The sole author designed, analysed, interpreted and prepared the manuscript.

Article Information

DOI: 10.9734/ARRB/2020/v35i330215

Editor(s):

(1) Paola Angelini, University of Perugia, Italy.

Reviewers:

(1) Maged Refaat, Ain Shams University, Egypt.

(2) Celso Eduardo Olivier, Instituto Alergoimuno de Americana, Brazil.

Complete Peer review History: http://www.sdiarticle4.com/review-history/57330

Original Research Article

Received 14 March 2020

Accepted 18 May 2020

Published 19 May 2020

\section{ABSTRACT}

Background: Type III hypersensitivity caused by immunoglobulin $G(\lg G)$ to food has elicited plenty of scholarly attention in recent years. Opinions continue to be divided regarding the linkage between IgG reactivity to food and chronic inflammatory diseases.

Objective: To identify foods that cause the maximum amount of IgG immune reaction in patients throughout Saudi Arabia from a standardized food panel and to identify any difference caused by age or gender.

Methods: We used a pre-existing database for patients who participated in the standardized panel of 268 foods ELISA-based IgG to conduct a food allergy test referred to as ImuPro ${ }^{\mathrm{TM}}$ Complete. The data in the database was prepared by the database provider through the utilization of established procedures from R-Biopharm AG using standard ELISA plates. Meanwhile, the samples were processed in ELISA Washer and Reader machines. Readings were analyzed using R-Biopharm's standard ImuPro ${ }^{\mathrm{TM}}$ software and then fed into the database used by us.

Results: A total of 1644 patients (913 males and 731 females $=55.5 \%$ male, $44.5 \%$ female) were tested. IgG reactivity was predominant in eight foods (3\%) in at least three-quarters of the studied population. These included Oats $(82.5 \%)$, Barley $(79.1 \%)$, Rye $(76.1 \%)$ Cow's Milk $(75 \%)$, Wheat $(74.9 \%)$, Kamut $(74.6 \%)$, Spelt $(74.6 \%)$ and Gluten $(73.9 \%)$. The IgG immune response of males and females to each food type was found to be almost the same. Children have a significantly different $\lg G$ food profile in comparison to adults ( $p$-value $=0.024$ ). Cow's milk was found to induce 
the highest IgG immune response among children below five years of age (95.74\%), followed by oats $(92.2 \%)$.

Conclusion: To the best of our knowledge, this is one of the largest studies to have been conducted worldwide that involves the $\mathrm{IgG}$ immune response of patients to food. The top-most foods were 'mainstream' foods consumed almost daily, which include gluten and dairy products. No significant difference was found between males and females. A clear difference can be seen between children and adults when it comes to the IgG immune reaction to different food items. Further investigations are recommended to determine the food characteristics and eating habits that cause these $\lg G$ immune responses.

Keywords: Chronic illness; chronic inflammation; food allergy; IBS; IgE; IgG; immunoglobulin E; immunoglobulin G; immunoglobulin.

\section{INTRODUCTION}

Allergy is known to affect millions of people worldwide. In this context, Immunoglobulin $\mathrm{E}$ $(\lg E)$ allergy, which is also known as type I allergy, has been extensively studied $[1,2,3,4]$. Allergies can be classified into two major groups: inhalant and food allergies. About $4-8 \%$ of the population suffers from food allergy $[4,5]$. There is a smaller subclass called "Monoallergy", which denotes allergy to different drugs and medications, including those for insect bites. Although this is an IgE-mediated hypersensitivity, the total $\lg E$ is not increased. This type of allergy can be acquired anytime during the life of the patient and is not known to have any inducing factors [1].

In addition to $\lg E$ mediated allergy, a second type of immune response may occur against food. This type of response is categorized as Type III IgG food sensitivity $[6,7,8]$, which has recently gained a lot of attention from the medical community. It is assumed to affect up to $20 \%$ of the population suffering from chronic inflammatory diseases. The IgG-mediated hypersensitivity is mostly a delayed-type of allergy, with symptoms usually appearing hours or up to three days after the consumption of concerned food $[9,10]$. This makes it practically impossible to identify such foods without testing them. The objective of this study is to determine the foods that caused the maximum IgG immune response in patients that underwent the ImuPro Complete test against all the 268 foods. In addition, it aims to examine whether there is any difference in the above response between males and females, and between children and adults. According to the findings, most of the foods causing this response are mainstream foods. For this reason, it is interesting to study the causes of this type immune response.
The presence of $\lg G$ against food is a contentious issue $[11,12]$ Some authors believe that it is normal to have IgG antibodies to food that are regularly consumed, and that the presence $\operatorname{lgG}$ is a marker of contact and tolerance, rather than hypersensitivity. However, this viewpoint has been contradicted by a study [13] carried out in Germany in which volunteers who were lgG negative to soy, cow's milk, or eggs were required to follow a 3-week provocation diet containing in addition to their daily intake: either $750 \mathrm{ml}$ of soy milk, $750 \mathrm{ml}$ of cow's milk, or two eggs. After four and seven weeks, IgG titers were measured, and none showed any elevated $\lg G$ to the consumed foods. Moreover, it is impractical for any individual to possess antibodies to all foods, including beef, chicken, rice, potatoes, and vegetables. It is noteworthy that less than $5 \%$ of people are allergic to beef, although millions of people globally consume beef once per week. Humans have developed a high degree of tolerance against food [14], which implies an absence of immune reaction. This is the normal situation. We only develop antibodies to a particular type of food when our immune systems react to it and tag it as something potentially dangerous. An IgG-mediated immune response is triggered every time someone consumes food that the body's IgG immune system considers as a threat.

Thousands of patients have undergone and benefitted from the IgG test. In general, these patients suffer from chronic medical conditions such as irritable bowel syndrome (IBS) [15,16], migraine [16,17], psoriasis, joint pains, Crohn's disease [18,19], hypertension [20], and asthma [21], to name a few. Many of them have even taken medical treatments without positive outcomes. In addition, a number of these patients carry on with their lives by taking medications that treat the symptoms but do not 
address the root cause of the problem. Food might not be the primary cause for their ailments, but IgG marked food antigens may indeed be fixed by a sensitized organ, attract phagocytes, and get destroyed locally. This action will inflame the tissue before increasing the progression of the disease. In order to stop this process, one needs to identify and remove individual triggers.

However, it is observed that patients who take the IgG test, either because their doctor advise them to do so, or because they stumble upon it while searching the internet for a cure to their pain and suffering, get cured of their problems by excluding these foods from their diet $[10,17]$.

IgE-mediated food allergy is a prevalent type of sensitivity reaction that can occur anytime or anywhere. These allergies are quite difficult to avoid and are usually treated by some medicines, such as antihistamines [22]. However, preventing these allergies may become difficult since they can occur due to everyday food items such as, milk, chicken, eggs, and peanuts [23]. These foods, along with many other common food items, can cause lifethreatening anaphylaxis $[24,25]$.

IgG food hypersensitivity occurs when the immune system recognizes food as a foreign invader. As a result, the immune system produces $\lg G$ antibodies that attack the foreign food items. The four subclasses of $\lg G$ are: $\lg G 1$, $\lg$ 2, IgG3 and IgG4. IgG1-3 are proinflammatory antibodies, while $\operatorname{lgG} 4$ has antiinflammatory properties $[26,27,28,29,30]$. IgG4 is regarded as the antidote to $\mathrm{IgE}$, as the liberation of histamine is strongly reduced, and allergic symptoms can be avoided. IgG4 is the type of antibody that is produced during desensitization of type 1 allergy. IgG4 cannot induce an allergy or inflammatory response, which is considered to be protective and induce tolerance. IgG4 only constitutes $5 \%$ of total IgG in serum and its concentration is much lower than the other subclasses. In the test used for this study, only $1 \%$ of the reactions can be attributed to $\lg 4$. In contrast IgG1-3 have strong pro-inflammatory properties, they can activate the complement system; they are opsonizing and induce chemotaxis, which results in the destruction of the formed immune complex by phagocytosis. The main white blood cells involved are neutrophils. In case of injected allergens, the mechanism leading to $\mathrm{IgG}$ mediated immune reactions and anaphylaxis were published by Jönsson et al. [31]. They found that neither IgE, histamine, basophiles, normastocytes were involved, but that $\lg G$, IgG receptors, and neutrophils were responsible for the observed anaphylaxis. Furthermore, PAF (platelet activating factor) was observed to be one of the most potent cytokines involved. As observed in this study, food is ingested instead of being injected, which leads to the destruction of epitopes, a dilution and delayed uptake from the gut to the blood. For these reasons, IgG reaction to food doesn't lead to anaphylaxis or immediate reaction such as $\lg E$. Instead, it causes the onset of delayed milder symptoms. This inflammatory or hypersensitivity reaction, in turn, may produce various other symptoms such as constipation, tiredness, bloating, irritable bowel syndrome (IBS), diarrhea, migraines, eczema, and headaches [32,33].

It is notable that IgG I, IgG II, or IgG III reaction to certain kinds of food is found to occur every time the patient consumes the affecting food item. As a matter of fact, everyday consumption of these foods induces this inflammatory process on a daily basis. This is when the useful IgG reaction may become harmful since the immune system treats these foods as a threat in such cases and reacts accordingly.

Studies have shown that an increase of inflammation markers such as serum CRP, white blood cell counts, Calprotectin could be observed within a short time after consumption of IgG positive foods $[19,20]$.

There are several types of food testing, but the most common method is to test or screen the patient against an innumerable number of food additives and items. Consumers are then provided with a list of food items that they are intolerant to.

In this regard, IgG (immunoglobulin $\mathrm{G}$ ) testing is one of the most useful methods for food intolerance testing $[20,34,35]$. The test is used in various kinds of conditions where diet elimination and preventing allergies is deemed mandatory. Such conditions can include movement disorders, gastrointestinal, and neurological conditions [36]. IgG testing is not intended to confirm any diagnosis. Instead, it is used as a guide to identify the foods that cause elevated IgG levels in the blood. The next step is to follow the gold standard for diagnosing food allergy, which to eliminate these foods [37] from the diet followed by a provocation diet where these foods are reintroduced one at a time to determine the foods that actually cause the symptoms to reappear [38]. 
In order to resolve this problem, food specific $\lg G$ testing helps identify the food items that cause IgG hypersensitivity reactions before eliminating them from the individuals'dietary intake [8]. By doing so, a variety of medical conditions such as epilepsy, rheumatoid arthritis [39], cystic fibrosis, autism, $A D(H) D$, and IBS can disappear or be be effectively addresses $[15,40]$.

\section{METHODS}

For this study, a preexisting database for patients in Saudi Arabia underwent the ImuPro ${ }^{\mathrm{TM}}$ food allergy test between 2011 and 2016 was used. This test was devised by R-Biopharm AG from Germany and is an enzyme-linked immunosorbent assay (ELISA). It is used a standardized panel of 268 foods ELISA-based IgG food allergy test. The database provider had all the samples processed by CTL laboratory, Germany, and $5 \mathrm{ml}$ of blood was extracted from each patient, before being centrifuged and collecting the serum. Standard procedures from R-Biopharm AG using standard ELISA plates for performing the ImuPro ${ }^{\mathrm{TM}}$ food allergy test were then adhered to. In addition, all the samples were sent across to CTL laboratories in Germany with a view to processing the samples in an ELISA Washer and Reader machines. The measurements were analyzed by R-Biopharm's standard ImuPro ${ }^{\mathrm{TM}}$ software and the results were put in the database used by us.

Only numerical test results, as well as patient age and gender data, were used in this study. No patient identifying information was used. Inclusion criteria included patients who were part of a pre-existing database and took the aforementioned test in Saudi Arabia between 2011 and 2016. A total of 1644 patients (913 males and 731 females of different ages) were analyzed. The large size of this sample made it possible to represent all patients and no exclusion were made. Some patients were referred by physicians, while others opted to perform the test on their own accord. In other words, the sample was representative of all patients.

Patients signed a consent form, which allowed their data to be used for research purposes.

\section{RESULTS AND DISCUSSION}

This section explains the findings based on actual measurements involving 1644 patients
(913 males and 731 females) of all ages ranging from 1 year up to 80 years old in Saudi Arabia who were tested for the same 268 food types.

Fig. 1 shows the topmost food groups that induced an IgG immune response. The size of the circle indicates the percentage of the population affected by this food group. Evidently, milk products, eggs, yeast, cereals, and seeds emerged as the biggest contributors to the $\lg G$ immune response.

Fig. 2 (a) uses a Box and Whisker plot to display the distribution of foods, thus inducing an IgG immune response in the population tested. As shown by the above figure, the majority of foods ( 243 foods $=91 \%$ ) tested affected less than $30 \%$ of the population. In addition, it can also be seen that 25 outliers affect more than $70 \%$ of the population. Fig. 2(b) shows the outliers and the percentage of the population affected.

Shakoor et al. [9] performed a similar study using a microarray test and tested 223 food types. Their study involved 71 patients (49 males and 22 females) between the ages of 6 and 80 years. They excluded patients with IgE symptoms and elevated IgE levels, as well as those with positive skin prick test and any disorders other than allergies including IBS, food enzyme deficiency, and celiac disease. Our study is different in that we intentionally did not have any exclusion criteria to obtain a global view of the society lgG profile. We included 1-year old children and older, used an ELISA test, tested 268 different food types, and had 1644 patients (913 males and 731 females). Meanwhile, our panel includes several types of beans (cocoa bean, green bean, soybean, broad bean, and mung bean), but excludes cola bean and red kidney beans. It is interesting to compare some of their results to ours for similar foods, as shown in Table 1. It can be seen that we have a close match for wheat and egg white. The difference in results is actually quite interesting and opens up further research opportunities to investigate the underlying reason for these differences, be it due to the age difference, the exclusion criteria used by Shakoor et al. [9], the different sensitivities of microarray and ELISA, or some other factors. Another reason for the difference between our results and that of Shakoor et al. [9] is that our study used different test technologies from different companies, which could include different sources of antigens and a unique chemistry behind each testing system. 


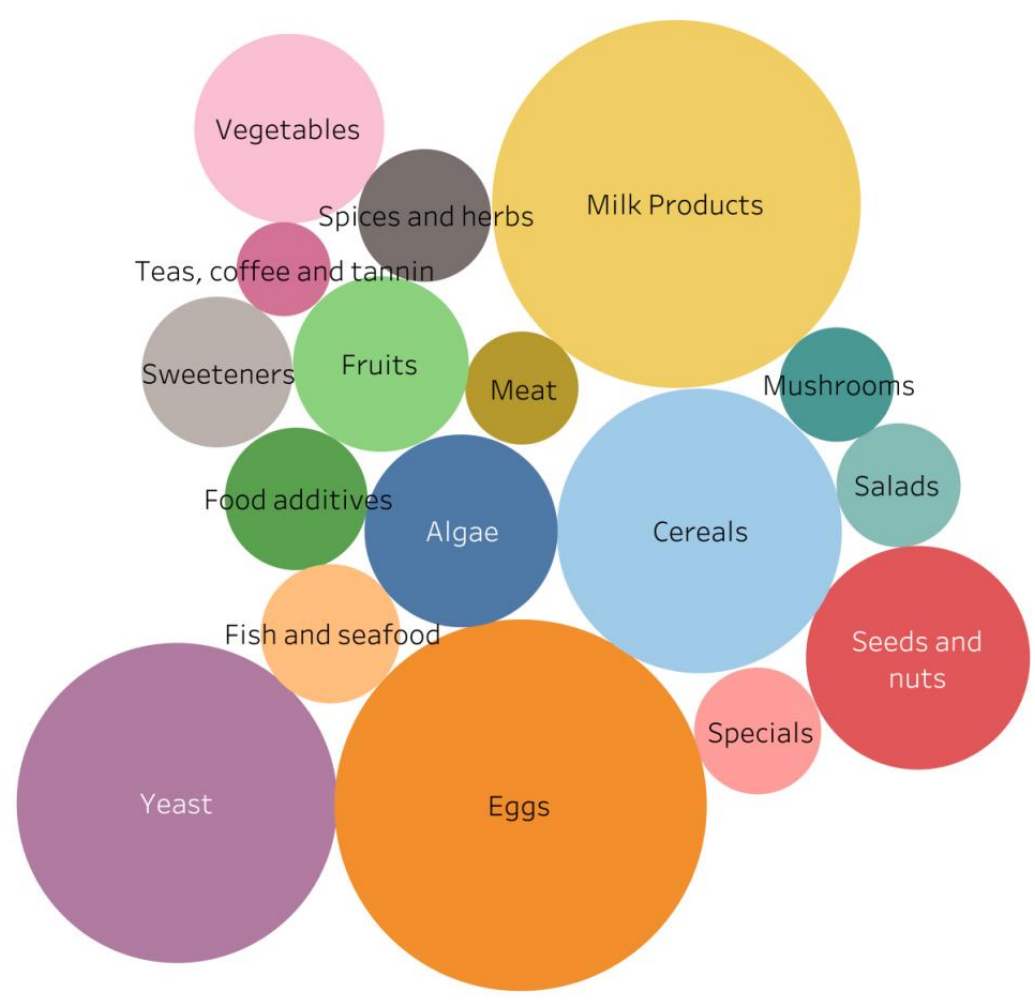

Fig. 1. Food groups causing IgG immune reaction. The size of the circle indicates the percentage of the population affected by this food group

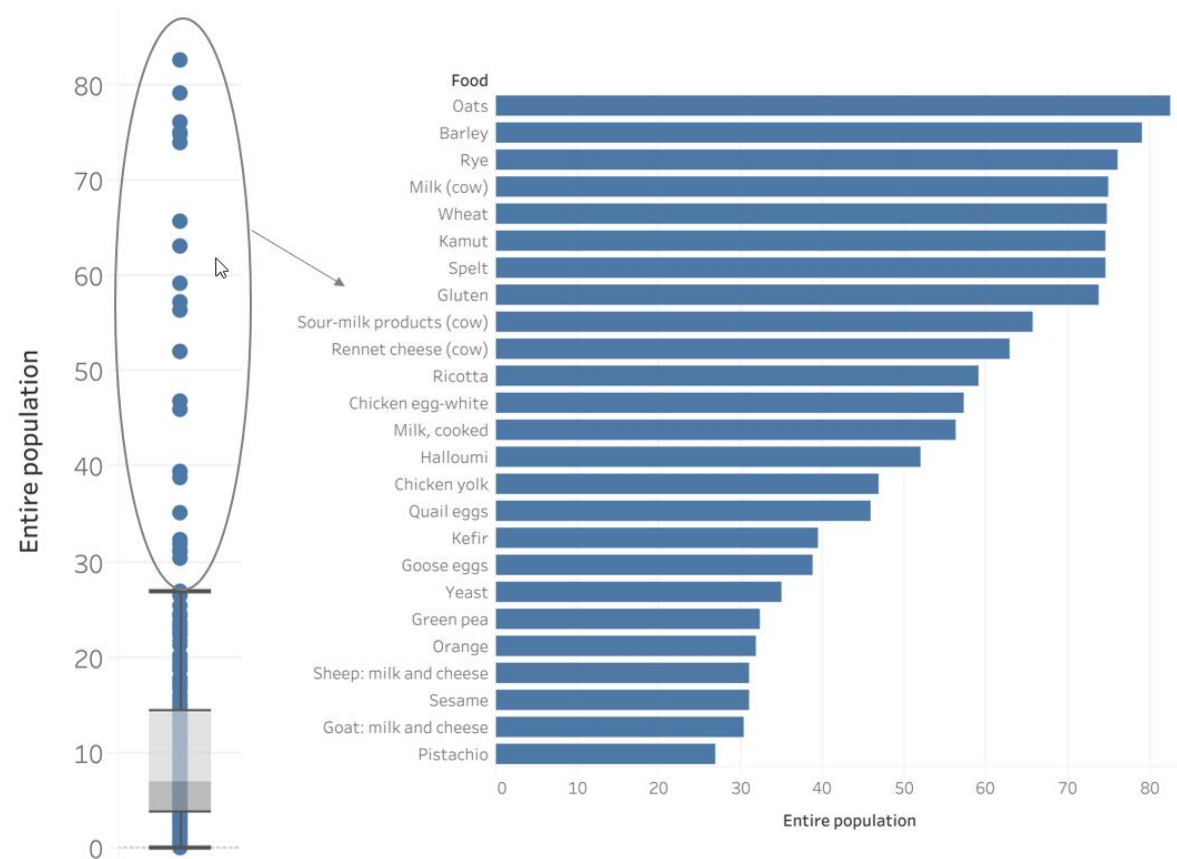

(a)

(b)

Fig. 2(a) Box and Whisker plot of the entire data with outliers. (b) Outlier foods with the percentage of the population they affected 
Table 1. Comparison with the results of Shakoor et al. [9]

\begin{tabular}{lll}
\hline Food tested & Our results & Shakoor et al. [9] \\
\hline Yeast & $35.0 \%$ & $78.9 \%$ \\
Wheat & $74.9 \%$ & $77.5 \%$ \\
Pea & $32.4 \%$ & $63.4 \%$ \\
Corn & $10.6 \%$ (maize) & $62.0 \%$ \\
Egg white & $52.3 \%$ & $62.0 \%$ \\
Barley & $79.1 \%$ & $57.7 \%$ \\
Pistachio & $26.9 \%$ & $56.3 \%$ \\
Cow's milk & $75.0 \%$ & $56.3 \%$ \\
\hline
\end{tabular}

Fig. 3 (a) illustrates the same data shown in Fig. 2(a), albeit in a histogram plot. It plots the percentage of patients affected by each food type. For analogy purposes, the curve shown in Fig. 3(a) denotes an exponential probability density random function. We can determine the probability characteristics for any region by means of probability rules.

Fig. 3(b) shows the foods affecting over $50 \%$ of the population. The size of the circle reflects the percentage of the population affected. A comparison between the data in Fig. 2(b) and Fig. 3(b) clearly reveals the presence of a well-defined prevalence of IgG allergic response to foods. Meanwhile, Table 2 shows that 26 foods $(9.7 \%$ of the foods tested) caused an IgG reaction in $26 \%$ of the population, whereas 14 foods $(5.2 \%$ of the foods tested) triggered an IgG reaction in $51 \%$ of the population. However, only eight foods (3\% of the foods tested) were found to trigger an IgG immune response in more than $73 \%$ of the tested patients.

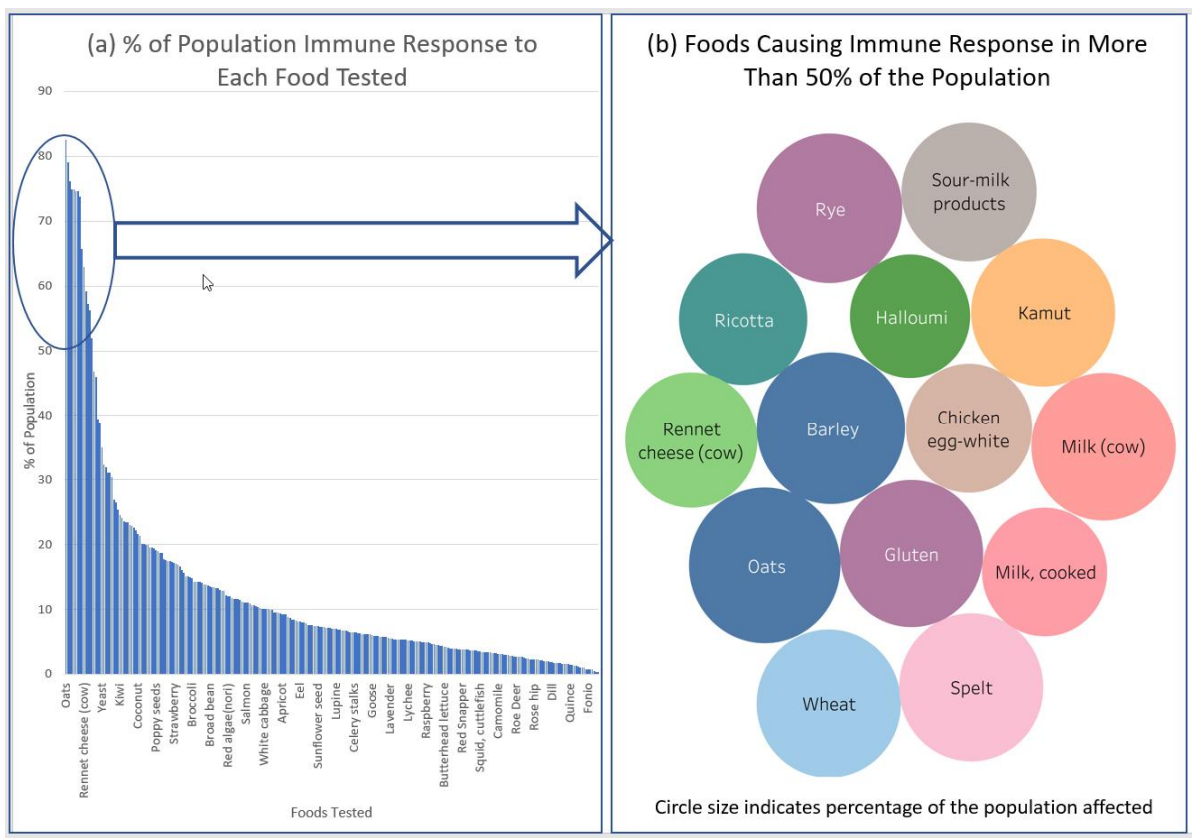

Fig. 3. \% of patients with IgG levels above the cut-off threshold for each food tested in descending order

Table 2. Number of foods causing the most IgG immune response

\begin{tabular}{lll}
\hline \# of Foods & \% Compared to total foods tested & \% of Population affected \\
\hline 26 & $26 / 268=9.7 \%$ & $>26.5 \%$ \\
14 & $14 / 268=5.2 \%$ & $>51.2 \%$ \\
8 & $8 / 268=3.0 \%$ & $>73.8 \%$ \\
\hline
\end{tabular}


Fig. 2(b) shows that the outlier foods exhibit an overlapping trait:these 'mainstream' foods are consumed by the majority of people almost on a daily basis, forming part of the majority of our diets. These primarily include wheat, gluten, cow's milk, and eggs. This is further clarified if we study Fig. 3(b) where it can be seen that the 14 foods affecting more than $50 \%$ of the population are actually mainstream foods consumed on a regular basis. It is also pertinent to look at the other end of the spectrum and analyze the ten food items that cause the least IgG allergic response in the population tested.

Table 3 lists ten foods causing the least $\lg G$ response in the descending order. Notably, although cow's milk affects $75 \%$ of the population and is consumed directly or indirectly on a daily basis as an ingredient used in various food recipes, there are other foods that are consumed almost as frequently, but induce almost negligible IgG immune response. For example, our results show that black tea and green tea have a very low effect on IgG immune response, affecting only $1.58 \%$ and $0.72 \%$ of the participants, respectively. Although people drink tea and cow's milk almost on a daily basis, the former impacts less than $2 \%$ of the population tested, while the latter affects $75 \%$ of the population tested. Some of the other foods that are consumed almost every day include chicken, beef, and rice; however, they impact only $11.98 \%, 5.42 \%$, and $4.89 \%$ of the population, respectively. Thus, it can be concluded that merely eating a particular food on a regular basis and in large quantities alone is not a strong enough reason to cause a large percentage of the population to develop an IgG immune response to it. It is important for the foods to have some intrinsic properties that cause many people to develop an IgG immune response to these items when consumed frequently and in large quantities. This point is an interesting topic for further research.

Since all the patients were tested for the same 268 food items, their median is 134 foods. The five foods that fall above and below the median are depicted in Table 4. It is evident that most foods around the medial induced an IgG response in only about $7 \%$ of the population tested.

Table 3. Ten foods causing the least response in the population

\begin{tabular}{lll}
\hline & Food & \% of Population \\
\hline 1 & Tannin & 1.07 \\
2 & Lemon balm & 1.07 \\
3 & Teff & 1.07 \\
4 & Fonio & 0.77 \\
5 & Carrageenan (E407) & 0.77 \\
6 & Goat meat & 0.72 \\
7 & Tea, green & 0.72 \\
8 & Nettle & 0.42 \\
9 & Moluchia & 0.36 \\
10 & Saffron & 0.30 \\
\hline
\end{tabular}

Table 4. Ten foods around the median

\begin{tabular}{lll}
\hline & Food & \% of Population \\
\hline 129 & Rosemary & 7.33 \\
130 & Bamboo shoots & 7.15 \\
131 & Sunflower seed & 7.39 \\
132 & Sea bass & 7.09 \\
133 & Thyme & 7.21 \\
134 & Amaranth & 7.15 \\
135 & Cauliflower & 6.97 \\
136 & Lupine & 6.97 \\
137 & Pectin (E440) & 6.85 \\
138 & Oysters & 6.73 \\
139 & Nutmeg & 6.79 \\
\hline
\end{tabular}


It is also interesting to compare the results of male and female participants. Fig. 4 illustrates the percentage of males and females who reported an IgG immune response to each food tested. It was observed that the majority of these foods induced almost the same effect on both males and females.

Fig. 5 plots the percentage of population affected by each food for children below the age of five against the percentage of the population above the age of 15 years that were affected by the same food. An analysis of these foods reveals a clear difference. Some foods were found to affect the majority of children below five years much more than the remainder of the population above the age of 15 . While other foods were observed to have a minor effect on children below the age of five, they had a stronger impact on the remainder of the population above 15 years of age. In general, Fig. 5 demonstrates that children are affected by more types of foods than adults. This becomes evident from the prevalence of more data points to the right-hand side of the 45degree line.

Fig. 6(a) illustrates a Box and Whisker plot to display each food and subtracts the percentage of the children population below the age of five from the percentage of the remainder of the population above 15 years of age who developed an IgG immune response to that same food. It can be seen that there are 22 foods in the upper outlier (foods that affected children much more than adults) and there are three foods in the lower outlier are listed in Fig. 6(b). This clearly shows that a major change occurs in the immune profile of children and adults. For example, $60 \%$ more children below the age of five years showed an IgG immune reaction to coconut as compared to adults older than 15 years. Contrastingly, more than $30 \%$ of adults reported an IgG immune response to black pepper in comparison to children below the age of five.

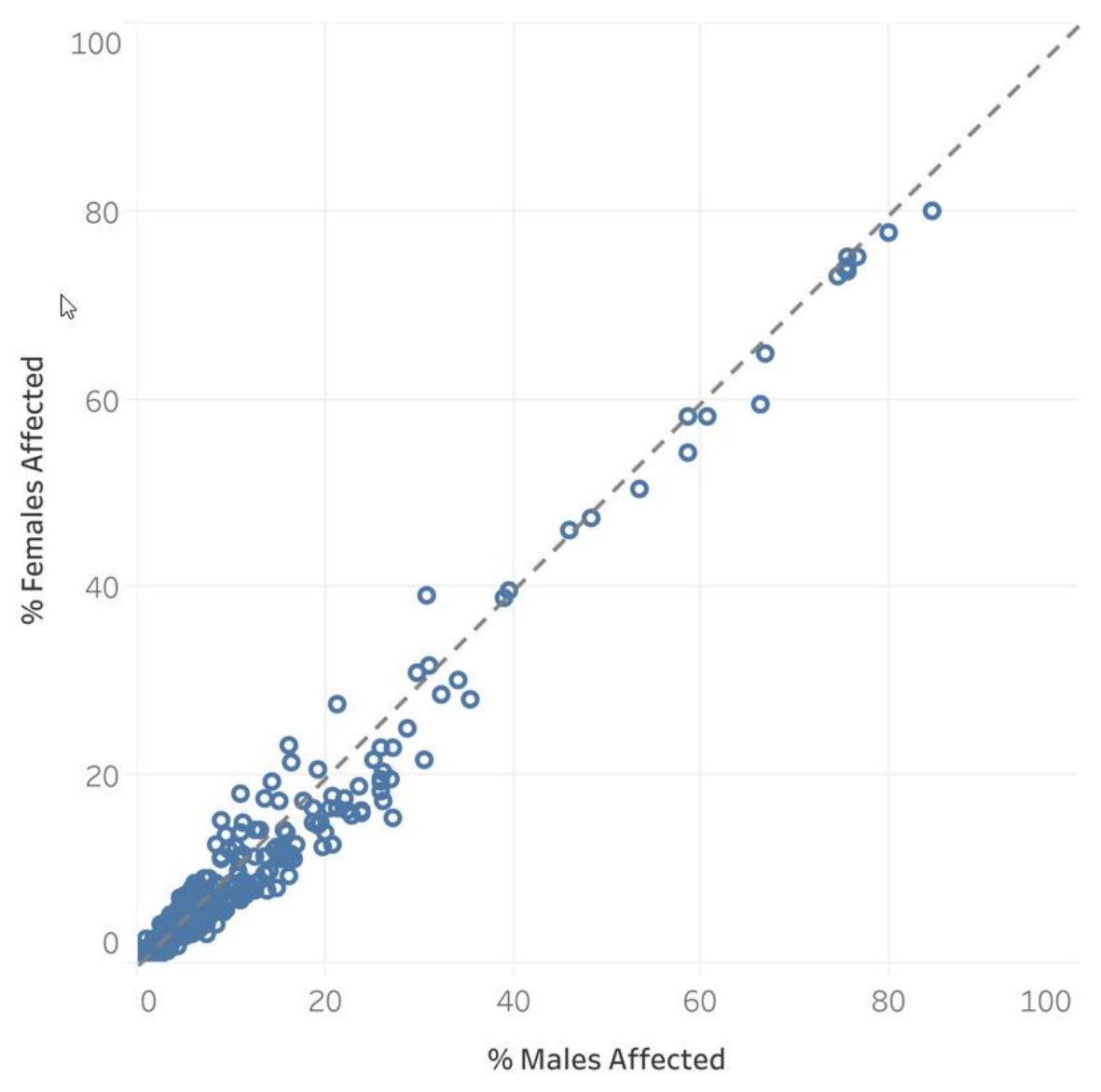

Fig. 4. Comparing male and female Immune response to all foods tested 


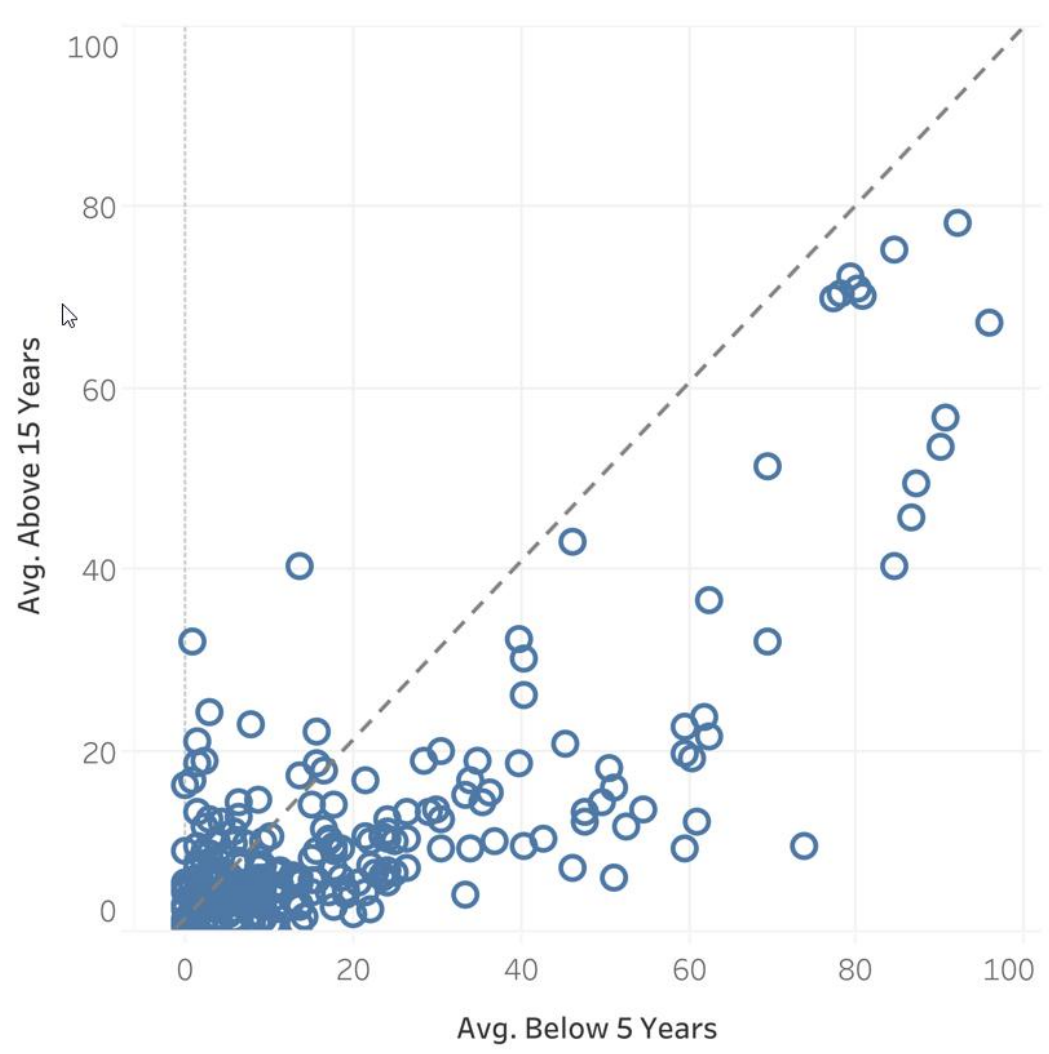

Fig. 5. Comparing IgG induced immune response for each food in children below five years old and the remainder of the population above 15 years of age

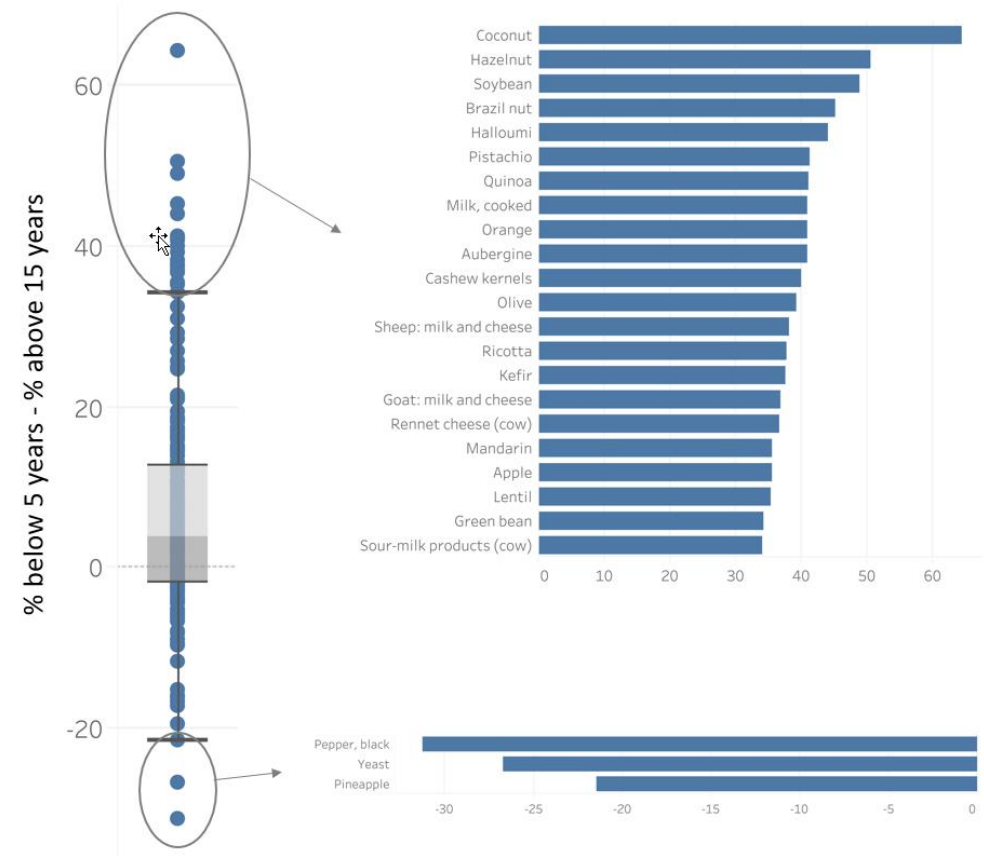

Fig. 6(a). Box and Whiskers plot showing for each food tested the \% affected children below five years - $\%$ affected for those above 15 years, (b) outliers 
Table 5. List of foods where the difference between the $\%$ affected Adults and children was the greatest

\begin{tabular}{llll}
\hline Food & $\begin{array}{l}\text { \% Affected } \\
\text { below five years }\end{array}$ & $\begin{array}{l}\text { \% Affected } \\
\text { above 15 years }\end{array}$ & Difference \\
\hline Coconut & 73.76 & 9.45 & 64.31 \\
Hazelnut & 59.57 & 9.11 & 50.46 \\
Soybean & 60.99 & 12.11 & 48.88 \\
Brazil nut & 51.06 & 5.93 & 45.13 \\
Halloumi & 84.4 & 40.38 & 44.02 \\
Pistachio & 60.28 & 18.99 & 41.29 \\
Quinoa & 54.61 & 13.49 & 41.12 \\
Milk, cooked & 86.52 & 45.62 & 40.9 \\
Orange & 62.41 & 21.56 & 40.85 \\
Aubergine & 52.48 & 11.68 & 40.8 \\
Cashew kernels & 59.57 & 19.59 & 39.98 \\
Sheep: milk and cheese & 61.7 & 23.54 & 38.16 \\
Ricotta & 87.23 & 49.57 & 37.66 \\
Kefir & 69.5 & 31.96 & 37.54 \\
Goat: milk and cheese & 59.57 & 22.68 & 36.89 \\
Rennet cheese (cow) & 90.07 & 53.44 & 36.63 \\
Apple & 49.65 & 14.18 & 35.47 \\
Lentil & 51.06 & 15.81 & 35.25 \\
Sour-milk products (cow) & 90.78 & 56.7 & 34.08 \\
Pepper, black & 0.71 & 31.96 & -31.25 \\
Yeast & 13.48 & 40.21 & -26.73 \\
Pineapple & 2.84 & 24.31 & -21.47 \\
\hline
\end{tabular}

Table 5 Compares the foods that caused an IgG immune response in $50 \%$ or more in the children's population below the age of five (141 children), as well as the corresponding percentage of affected population above 15 years of age (1164 persons) to the same food. The data is sorted in a descending order for $\%$ of children affected.

In this analysis, we determined the allergic level of food items versus the individual's age, starting from a very early age. Our results were compared based on the foods triggering an IgG immune response in children aged under five years (141 children compared with the rest of the population above 15 years of age (1164 persons). The selection of these age groups is intended to emphasize the role of age with regards to the IgG immune response. This approach facilitates an understanding of the nature of $\operatorname{lgG}$ food Immune response in human beings. Table 5 shows different results for both children and adults. The most interesting result seen in the table is that cow's milk has the highest indication of IgG response for children below five years of age. This food item is followed by oats, cow sour-milk, rennet cheese, ricotta cheese, and cooked milk, respectively. However, the order and prevalence of the same foods on individuals above the age of 15 years for the same foods was observed to change. This result leads to the conclusion that the foods derived from cow's milk induce the strongest IgG allergic response in children below five years of age. This effect continues to reduce with age.

\section{CONCLUSION}

This study obtained and compared the results from 1644 patients in Saudi Arabia who underwent the ELISA-based ImuPro IgG test (from R-Biopharm AG - Germany) on 268 food items. According to the findings, eight foods representing $3 \%$ of the tested foods affected $73.4 \%$ of the population. Notably, all the topmost type III IgG allergy-causing foods are essentially 'mainstream' foods that are consumed by the majority of people almost on a daily basis. They primarily comprise of wheat, gluten, cow's milk, and eggs. Contrastingly, other common foods such as beef, and rice and drinks such as tea, elicited very little IgG response in the population. Foods were also found to affect both males and females almost equally.

Most crucially, the most significant foods for children below the age of five include cow's milk, oats, sour cow's milk, rennet cheese, ricotta 
cheese, and cooked milk. For those older than 15 years, the sequence of the same food items is $8,1,9,10,12$, and 13 , respectively. Thus, it can be inferred that cow's milk causes the strongest IgG allergic response in children younger than five years of age, because children have a significantly different $\lg G$ food profile as compared to adults $(p<.05$ i.e. $p$-value $=.024$ ). With the increase in age, a gradual change is observed in the foods that cause IgG allergic responses.

In summation, it can be concluded that IgG induced immune response for some foods can be eradicated or reduced drastically with time. At the same time, the IgG induced immune response for some foods can appear or even radically increase with time. These findings reinforce the belief that the adverse of type III IgG food immune response can be reduced or even completely cured, and that the method used for testing $\lg G$ immune response is far more effective than traditional methods. These results are valid for Saudi Arabia, and may vary from one continent to another due to cultural and/or genetic factors. It would be interesting to conduct further investigations provided the same testing system is used to compare the results.

\section{ACKNOWLEDGEMENT}

The author would also like to express his gratitude to Dr. Khaled Daqrouq (Biomedical Engineering Specialty, Electrical and Computer Engineering Department, Faculty of Engineering), Prof. Emad Koshak (Allergy and Immunology Division, Internal Medicine Department, Faculty of Medicine) and Dr. Mazin Alahmadi (Industrial Engineering Department, Faculty of Engineering), all of whom are from King Abdulaziz University, Jeddah, Saudi Arabia, for their valuable time, comments, encouragement, and support. The author would also like to thank Dr. Camille Liners for his insightful discussions and discussions.

\section{COMPETING INTERESTS}

Author has declared that no competing interests exist.

\section{REFERENCES}

1. Olivier CE. Food allergy. J Aller Ther S. 2013;3:2.

2. Akdis CA, Blaser K, Akdis M. Apoptosis in tissue inflammation and allergic disease.
Current Opinion in Immunology. 2004; 16(6):717-723.

3. Branum AM, Lukacs SL. Food allergy among US children: Trends in prevalence and hospitalizations; 2008.

4. Gupta RS et al. The prevalence, severity, and distribution of childhood food allergy in the United States. Pediatrics. 2011;128(1): e9-e17.

5. BB, JL, FG. Summary health statistics for U.S. Children: National Health Interview Survey, 2012. Vital Health Stat. Table 2, 2013;10:1-81.

6. RR. Coombs A. Classification of allergic reactions responsible for clinical hypersensitivity and disease. Clinical Aspects of Immunology; 1968.

7. SG. Johansson $O$. et al. A revised nomenclature for allergy: An EAACl position statement from the EAACl nomenclature task force. Allergy. 2001; 56(9):813-824.

8. Mullin GE, Swift KM, Lipski L, Turnbull LK, Rampertab SD. Testing for food reactions: The good, the bad, and the ugly. Nutrition in Clinical Practice. 2010;25(2):192-198.

9. Shakoor Z, Al Faifi A, Al Amro B, Al Tawil $L N$, Al Ohaly RY. Prevalence of IgGmediated food intolerance among patients with allergic symptoms. Annals of Saudi Medicine. 2016;36(6):386-390.

10. Guo H, Jiang T, Wang J, Chang Y, Guo H, Zhang $W$. The value of eliminating foods according to food-specific immunoglobulin $G$ antibodies in irritable bowel syndrome with diarrhoea. Journal of International Medical Research. 2012;40(1):204-210.

11. Stapel SO, et al. Testing for IgG4 against foods is not recommended as a diagnostic tool: EAACI Task Force Report. Allergy. 2008;63(7):793-796.

12. Carr S, Chan E, Lavine E, Moote W. CSACI position statement on the testing of food-specific IgG. Allergy, Asthma \& Clinical Immunology. 2012;8(1):12.

13. Rusch K, IgG-Diagnostik: Kein physiologischer Titeranstieg nach Lebensmittelverzehr. Medical special. 2010;1:29-30.

14. Chinthrajah RS, Hernandez JD, Boyd SD, Galli SJ, Nadeau KC. Molecular and cellular mechanisms of food allergy and food tolerance. Journal of Allergy and Clinical Immunology. 2016;137(4):984-997.

15. Atkinson $W$, Sheldon TA, Shaath $N$, Whorwell PJ. Food elimination based on $\lg G$ antibodies in irritable bowel syndrome: 
A randomised controlled trial. Gut. 2004; 53(10):1459-1464.

16. Aydinlar El. et al. IgG-based elimination diet in migraine plus irritable bowel syndrome. Headache: The Journal of Head and Face Pain. 2013;53(3):514-525, 2013.

17. Alpay K, Ertaş M, Orhan EK, Üstay DK, Lieners C, Baykan B. Diet restriction in migraine, based on IgG against foods: A clinical double-blind, randomised, crossover trial. Cephalalgia. 2010;30:7:829-837.

18. Bentz S. et al. Clinical relevance of $\operatorname{lgG}$ antibodies against food antigens in Crohn's disease: a double-blind cross-over diet intervention study. Digestion. 2010;81(4): 252-264.

19. Uzunismail $H$. et al. The effects of provocation by foods with raised $\lg G$ antibodies and additives on the course of Crohn's disease: A pilot study. The Turkish Journal of Gastroenterology: The Official Journal of Turkish Society of Gastroenterology. 2012;23(1):19-27.

20. Wilders-Truschnig $M$, Mangge $H$, Lieners C, Gruber HJ, Mayer C, März W. IgG antibodies against food antigens are correlated with inflammation and intima media thickness in obese juveniles. Experimental and Clinical Endocrinology \& Diabetes. 2008;116(04):241-245, 2008.

21. Vance GHS, Thornton CA, Bryant TN, Warner JA, Warner JO. Ovalbumin-specific immunoglobulin $\mathrm{G}$ and subclass responses through the first 5 years of life in relation to duration of egg sensitization and the development of asthma. Clinical \& Experimental Allergy. 2004;34(10):15421549.

22. Dannaeus $A$, Inganäs $M$. A follow-up study of children with food allergy. Clinical course in relation to serum IgE-and IgG-antibody levels to milk, egg and fish. Clinical \& Experimental Allergy. 1981; 11(6):533-539.

23. Rowntree S, Cogswell JJ, Platts-Mills TA, Mitchell EB, Development of $\lg$ E and $\lg G$ antibodies to food and inhalant allergens in children at risk of allergic disease. Archives of Disease in Childhood. 1985;60(8):727735.

24. Hunter JO. Food elimination in IBS: The case for IgG testing remains doubtful. Gut, 2005;54(8):1203-1203.

25. Sicherer SH. Food allergy: Practical diagnosis and management. CRC Press; 2013.
26. Vidarsson G, Dekkers G, Rispens T. IgG subclasses and allotypes: From structure to effector functions. Frontiers in Immunology. 2014;5:520.

27. Van der Zee JS, Van Swieten P, Aalberse RC. Inhibition of complement activation by IgG4 antibodies. Clinical and Experimental Immunology. 1986;64(2):415.

28. Aalberse RC, Stapel SO, Schuurman J, Rispens T. Immunoglobulin G4: An odd antibody. Clinical \& Experimental Allergy. 2009;3994:469-477.

29. Sampson HA. Food allergy. Part 2: Diagnosis and management. Journal of Allergy and Clinical Immunology. 1999; 103(6):981-989.

30. Ebisawa M, Ballmer-Weber BK, Vieths S, Wood RA. Food allergy: Molecular basis and clinical practice. Karger Medical and Scientific Publishers; 2015.

31. Jönsson $F$ et al. Mouse and human neutrophils induce anaphylaxis. The Journal of Clinical Investigation. 2011; 121(4):1484-1496, 2011.

32. Beyer K, Teuber SS. Food allergy diagnostics: Scientific and unproven procedures. Current Opinion in Allergy and Clinical Immunology. 2005;5(3):261266.

33. Skypala I, Venter C. Food hypersensitivity: Diagnosing and managing food allergies and intolerance. John Wiley \& Sons; 2009.

34. Arroyave Hernández $\mathrm{CM}$, Pinto $\mathrm{ME}$, Hernández Montiel HL. Food allergy mediated by IgG antibodies associated with migraine in adults. Revista Alergia de Mexico. 2007;54(5).

35. Park Mi, Camilleri M. Is there a role of food allergy in irritable bowel syndrome and functional dyspepsia? A systematic review. Neurogastroenterology \& Motility. 2006; 18(8):595-607.

36. Dannaeus A, Johansson SGO, Foucard T. Clinical and immunological aspects of food allergy in childhood. Acta Pædiatrica. 1978;67(4):497-504.

37. Dixon HS. Treatment of delayed food allergy based on specific immunoglobulin G RAST testing. Otolaryngology-Head and Neck Surgery. 2000;123(1):48-54.

38. Drisko J, Bischoff B, Hall M, McCallum R. Treating irritable bowel syndrome with a food elimination diet followed by food challenge and probiotics. Journal of the American College of Nutrition. 2006;25(6): 514-522. 
39. Li J. et al. The pathogenesis of rheumatoid arthritis is associated with milk or egg allergy. North American Journal of Medical Sciences. 2016;8(1):40.
40. Sicherer SH, Sampson HA. Food allergy. Journal of Allergy and Clinical Immunology. 2010;125(2):S116S125.

(c) 2020 Alkhateeb; This is an Open Access article distributed under the terms of the Creative Commons Attribution License (http://creativecommons.org/licenses/by/4.0), which permits unrestricted use, distribution, and reproduction in any medium, provided the original work is properly cited.

Peer-review history:

The peer review history for this paper can be accessed here: http://www.sdiarticle4.com/review-history/57330 